\title{
A cirurgia precoce nas fraturas do fêmur proximal em idosos reduz a taxa de mortalidade?*
}

\section{Does Early Surgical Fixation of Proximal Femoral Fractures in Elderly Patients Affect Mortality Rates?}

\author{
Igor Pellucci Pinto ${ }^{1}$ Luis Felipe Brandt Ferres ${ }^{1}$ Guilherme Boni ${ }^{1} \quad$ Guilherme Guadagnini Falótico ${ }^{1}$ \\ Maurício de Moraes $^{2}$ Eduardo Barros Puertas ${ }^{1}$ \\ ${ }^{1}$ Departamento de Ortopedia e Traumatologia, Escola Paulista de \\ Medicina, Universidade Federal de São Paulo (Unifesp), São Paulo, SP, Brasil \\ ${ }^{2}$ Serviço de Ortopedia, Hospital Municipal de Clínicas de São \\ Bernardo do Campo, São Bernardo do Campo, SP, Brasil \\ Rev Bras Ortop 2019;54:392-395. \\ Endereço para correspondência Igor Pellucci Pinto, MD, \\ Departamento de Ortopedia e Traumatologia, Escola Paulista de \\ Medicina, Universidade Federal de São Paulo (Unifesp), Rua Sena \\ Madureira 1.500, Vila Clementino, São Paulo, SP, 04021-001, Brasil \\ (e-mail: igor.pellucci@gmail.com).
}

\section{Resumo \\ Palavras-chave \\ - fraturas do quadril \\ - mortalidade \\ - idoso \\ - ortopedia}

\section{Abstract}

Objetivo Analisar se a redução no tempo de espera para cirurgia ortopédica nos pacientes idosos com fratura da extremidade proximal do fêmur impacta na redução da mortalidade intra-hospitalar, da mortalidade em 6 meses de seguimento, e na redução do tempo de internação hospitalar.

Métodos Trabalho de coorte retrospectiva fundamentado na análise de prontuários de 81 pacientes internados com diagnóstico de fratura transtrocanteriana ou subtrocanteriana do fêmur, e submetidos a tratamento cirúrgico em 2015 e 2016 em um hospital de referência no atendimento ao trauma, antes e após a implantação de protocolo para cirurgia em até 48 horas.

Resultados Observou-se redução do tempo médio de internação de 17 para 11 dias após a implantação do protocolo. Com relação à mortalidade intra-hospitalar, cinco pacientes faleceram antes da implantação do protocolo, e cinco, após a implantação do protocolo. Referente à mortalidade extra-hospitalar, avaliada em 6 meses de pósoperatório, observou-se uma redução de $26,7 \%$ para $19,4 \%$. Os resultados não apresentaram significância estatística.

Conclusão O presente estudo demonstrou que existe uma tendência à redução do tempo de internação hospitalar e da mortalidade em 6 meses quando a cirurgia para tratamento de fraturas do fêmur proximal no idoso é feita em até 48 horas de internação hospitalar.

Objective To analyze whether a reduction in the waiting time for orthopedic surgery in elderly patients with fracture of the proximal end of the femur leads to a reduction in in-hospital mortality, 6-month mortality, and duration of hospitalization.

\footnotetext{
Trabalho desenvolvido no Departamento de Ortopedia e Traumatologia, Escola Paulista de Medicina, Universidade Federal de São Paulo, São Paulo, SP, Brasil. Publicado originalmente por Elsevier Editora Ltda.

DIgor Pellucci Pinto's ORCID is https://orcid.org/0000-0003-4018-8127.
}

recebido

15 de Setembro de 2017 aceito

07 de Dezembro de 2017
DOI https://doi.org/

10.1016/j.rbo.2017.12.015. ISSN 0102-3616.
Copyright $\odot 2019$ by Sociedade Brasileira License terms de Ortopedia e Traumatologia. Published by Thieme Revnter Publicações Ltda, Rio de Janeiro, Brazil 


\section{Keywords \\ - hip fractures \\ - mortality \\ - elderly \\ - orthopedics}

Methods This was a retrospective cohort study including 81 patients with intertrochanteric and subtrochanteric fractures who underwent surgical repair between 2015 and 2016 in a referral hospital, before and after a protocol for early surgical fixation ( $<48$ hours) was implemented.

Results The mean length of hospital stay decreased from 17 to 11 days. Regarding the in-hospital mortality rate, five patients died before, and another five died after the protocol was implemented. The 6-month postoperative mortality decreased from $26.7 \%$ to $19.4 \%$. The results showed no statistical significance.

Conclusion The present study demonstrated that there is a tendency to reduce the length of hospital stay and 6-month mortality when the surgery for the treatment of proximal femoral fractures in the elderly is performed within 48 hours of hospitalization.

\section{Introdução}

Com o aumento progressivo da expectativa de vida observado nas últimas décadas, a prevalência do trauma no idoso também se elevou de forma significativa. A fratura de fêmur proximal é uma das fraturas mais comuns em idosos, e apresenta alta taxa de morbidade e de mortalidade, o que a torna um problema de saúde pública. Dados epidemiológicos apontam que a mortalidade em um ano de idosos com fratura do fêmur proximal varia de 14 a $36 \%,{ }^{1-6}$ e apenas 50 a $65 \%$ têm recuperação completa da atividade funcional prévia. ${ }^{1,7,8}$

Mais de $98 \%$ dos casos são tratados cirurgicamente, e evidências indicam que a cirurgia feita em até 48 horas reduz o risco de complicações secundárias à fratura do fêmur no idoso. Por outro lado, cirurgias feitas após 48 horas ou mais aumentam o risco de mortalidade em 30 dias e até 1 ano. ${ }^{9-13}$ Tendo em vista a importância dessa questão, desenvolveu-se o presente estudo com o objetivo de analisar se a redução no tempo de espera para cirurgia ortopédica nos pacientes idosos com fratura da extremidade proximal do fêmur impacta na redução da mortalidade intra-hospitalar, na mortalidade em 6 meses de seguimento, bem como no tempo de internação hospitalar.

\section{Material e Métodos}

Estudo de coorte retrospectiva, no qual foram selecionados pacientes com idade a partir de 60 anos, de ambos os sexos, com diagnóstico de fratura fechada, isolada, da região transtrocanteriana ou subtrocanteriana do fêmur. A classificação anatômica da fratura foi feita por um médico ortopedista com título de especialista no pronto-socorro, e confirmada pelo cirurgião ortopedista no hospital de retaguarda cirúrgica.

O presente estudo foi submetido e aprovado pelo Comitê de Ética em Pesquisa da nossa instituição, sob o parecer de número 1.890.165. Os dados referentes à internação hospitalar (tempo de internação, diagnóstico, tratamento, e desfecho clínico) foram obtidos por meio da revisão de prontuário eletrônico. A constatação do óbito e sua data foram obtidas por meio de informações no prontuário eletrônico, contato telefônico ou via obituário.
Elaborou-se, em 2016, um protocolo específico para o atendimento de pacientes idosos com fratura de fêmur proximal na rede de urgência e emergência do nosso hospital, a fim de se reduzir o tempo de espera para cirurgia ortopédica nesse tipo de fratura, além de reduzir a mortalidade e o tempo de internação. O objetivo primário desse protocolo é operar o paciente idoso com fratura transtrocanteriana e/ou subtrocanteriana em no máximo 48 horas após sua internação hospitalar.

Todos os pacientes do estudo foram internados e submetidos a tratamento cirúrgico em um hospital de referência no atendimento ao trauma a partir de 2015. Os pacientes foram divididos em 2 grupos: cirurgia dentro do protocolo de fratura de fêmur no idoso (após o primeiro trimestre de 2016), e cirurgia fora do protocolo (todo o ano de 2015 e o primeiro trimestre de 2016).

Foram excluídos pacientes que, durante a revisão dos prontuários, apresentavam dados incompletos e/ou insuficientes, pacientes submetidos a tratamento não operatório, e aqueles que não puderam ser contatados por telefone, que perderam acompanhamento.

As variáveis estudadas foram: tempo de internação (antes e após o protocolo), mortalidade geral, e mortalidade hospitalar e em até 6 meses pós-operatórios. Os dados foram analisados por meio de tabelas e dos testes estatísticos paramétricos de análise de variância (ANOVA, na sigla em inglês) e de hipótese de igualdade entre duas proporções, com o auxílio dos programas IBM SPSS Statistics for Windows, Versão 20.0 (IBM Corp., Armonk, NY, EUA), Minitab 16 (Minitab, LLC., State College, PA, EUA), e Excel (Microsof Corporation, Redmond, WA, EUA). Foi definido um nível de significância máximo de 5\% $(p \leq 0,05)$.

\section{Resultados}

Foram selecionados 159 pacientes internados em 2015 e 2016 sob o CID10 S72. Do total, 78 foram excluídos por não preencherem os critérios do estudo, o que resultou em uma amostra final de 81 pacientes ( $\mathbf{F i g .} \mathbf{1}$ ). Todos os pacientes tiveram seus prontuários eletrônicos revisados e/ou foram contatados por telefone. 


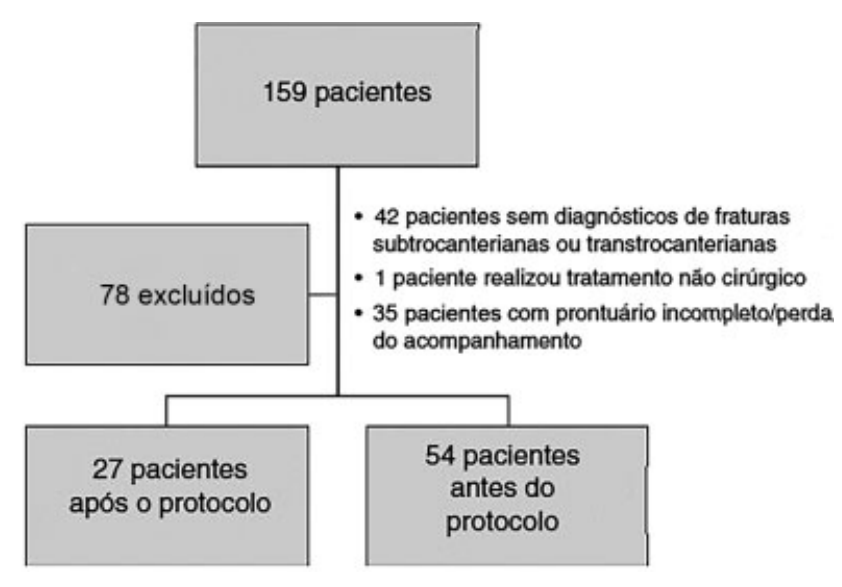

Fig. 1 Fluxograma de inclusão de pacientes.

O tempo médio de internação hospitalar foi de $\sim 17$ dias para o grupo avaliado antes da implantação do protocolo, e de 11 dias para os pacientes internados após a vigência do protocolo ( - Tabela 1). Não houve diferença estatisticamente significativa $(p=0,095)$.

Do total da amostra, 19 pacientes vieram a óbito durante o período de investigação, o que correspondeu a $26,7 \%$ do grupo fora, e a $19,4 \%$ do grupo dentro do protocolo, respectivamente $(p=0,446)$ ( - Tabela 2 ).

Durante a internação hospitalar, 5 pacientes no grupo fora e 5 pacientes no grupo dentro do protocolo faleceram; porém, não houve diferença estatística ao analisarmos ambos os grupos $(p=0,210)$ ( $($ Tabela 3 ).

\section{Discussão}

O presente estudo avaliou o tempo de internação e mortalidade intra-hospitalar e com 6 meses pós-operatórios em idosos que apresentaram fratura do fêmur proximal, e fez uma comparação entre pacientes incluídos e não incluídos em um protocolo elaborado com o objetivo de se fazer precocemente (em até 48 horas da internação) a cirurgia

Tabela 1 Comparação de dias de internação

\begin{tabular}{|l|l|l|}
\hline $\begin{array}{l}\text { Tempo de } \\
\text { internação } \\
\text { (dias) }\end{array}$ & $\begin{array}{l}\text { Antes da } \\
\text { implantação } \\
\text { do protocolo }\end{array}$ & $\begin{array}{l}\text { Depois da } \\
\text { implantação } \\
\text { do protocolo }\end{array}$ \\
\hline Média & 16,6 & 11,3 \\
\hline Mediana & 13 & 8 \\
\hline Desvio padrão & 14,8 & 9,3 \\
\hline $\begin{array}{l}\text { Coeficiente } \\
\text { de variação }\end{array}$ & $89 \%$ & $82 \%$ \\
\hline Valor mínimo & 6 & 2 \\
\hline Valor máximo & 108 & 35 \\
\hline N & 54 & 27 \\
\hline $\begin{array}{l}\text { Intervalo } \\
\text { de confiança }\end{array}$ & 4 & 3,5 \\
\hline Valor de $p$ & 0,095 & \\
\hline
\end{tabular}

Tabela 2 Mortalidade geral em seis meses

\begin{tabular}{|l|l|l|l|l|l|}
\hline & \multicolumn{2}{|l|}{$\begin{array}{l}\text { Antes da } \\
\text { implantação } \\
\text { do protocolo }\end{array}$} & \multicolumn{2}{|l|}{$\begin{array}{l}\text { Depois da } \\
\text { implantação } \\
\text { do protocolo }\end{array}$} & \multirow{2}{*}{ valor-p } \\
\cline { 2 - 5 } & $n$ & $\%$ & $n$ & $\%$ & \\
\hline Não óbito & 33 & $73,30 \%$ & 29 & $80,60 \%$ & 0,446 \\
\hline Óbito & 12 & $26,70 \%$ & 7 & $19,40 \%$ & \\
\hline
\end{tabular}

Tabela 3 Análise dos óbitos

\begin{tabular}{|l|l|l|l|l|l|}
\hline & \multicolumn{2}{|l|}{$\begin{array}{l}\text { Antes da } \\
\text { implantação } \\
\text { do protocolo }\end{array}$} & \multicolumn{2}{|l|}{$\begin{array}{l}\text { Depois da } \\
\text { implantação } \\
\text { do protocolo }\end{array}$} & \multirow{2}{*}{ valor-p } \\
\cline { 2 - 5 } & $n$ & $\%$ & $n$ & $\%$ & \\
\hline $\begin{array}{l}\text { Intra- } \\
\text { hospitalares }\end{array}$ & 5 & $41,70 \%$ & 5 & $71,40 \%$ & 0,21 \\
\hline Após a alta & 7 & $53,80 \%$ & 2 & $28,60 \%$ & \\
\hline
\end{tabular}

definitiva. Observamos a diminuição no tempo de internação e na taxa de mortalidade em 6 meses de pós-operatório, porém, sem significância estatística.

Nossos resultados mostraram uma redução da mortalidade geral em 6 meses de 26,7\% para 19,4\% após a vigência do protocolo (um valor elevado comparado a outros estudos, que pode ser explicado pelo pequeno número de pacientes analisados).

Embora seja preconizado que o tempo ideal para o tratamento cirúrgico da fratura do fêmur proximal seja 24 a 48 horas após a fratura, alguns estudos não demonstraram redução na mortalidade ou recuperação funcional com a cirurgia precoce. ${ }^{2,14-18}$

Orosz et al $^{2}$ estudaram 1.206 pacientes com mais de 50 anos, e obtiveram uma taxa de mortalidade geral em 2 meses de $8,3 \%$; após as análises, eles concluíram que a cirurgia dentro de 24 horas não reduz a mortalidade em 2 e 6 meses.

Siegmeth et al ${ }^{16}$ concluíram, após análise de 3.628 pacientes com mais de 60 anos (compensados clinicamente), que existe um aumento na mortalidade de pacientes previamente hígidos quando o procedimento cirúrgico é feito após 48 horas.

Em relação ao tempo de internação, notamos a redução de 17 para 11 dias, que, apesar de não apresentar associação significativa, é condizente com achados da literatura. Lefaivre et al $^{19}$ avaliaram 607 pacientes e concluíram que o atraso para fazer o tratamento cirúrgico aumentou o tempo de permanência hospitalar, e que estava relacionado a comorbidades médicas, idade, gênero e tipo de fratura.

Identificamos também um aumento percentual do óbito intra-hospitalar ao comparar os dois grupos do estudo, e isso possivelmente está associado às condições médicas do paciente e à menor amostra do grupo de pacientes operados após a implantação do protocolo. 
Como limitações do presente estudo, salientamos que se trata de uma análise retrospectiva, com uma casuística ainda pequena quando comparada com grandes estudos multicêntricos.

Estudos futuros serão feitos com aumento da população amostral após a continuidade do protocolo.

\section{Conclusão}

O momento ideal para o tratamento cirúrgico de fraturas do fêmur proximal em idosos ainda é controverso. Diversos fatores podem influenciar nessa decisão, tais como comorbidades clínicas do paciente, disponibilidade de material e de equipe treinada, bem como retaguarda de terapia intensiva para o pós-operatório. 0 presente estudo demonstrou que existe uma tendência à redução do tempo de internação hospitalar e da mortalidade em 6 meses quando a cirurgia para tratamento de fraturas do fêmur proximal no idoso é feita em até 48 horas de internação hospitalar.

Conflitos de Interesses

Os autores declaram não haver conflitos de interesses.

\section{Referências}

1 Zuckerman JD. Hip fracture. N Engl J Med 1996;334(23): 1519-1525

2 Orosz GM, Magaziner J, Hannan EL, et al. Association of timing of surgery for hip fracture and patient outcomes. JAMA 2004;291 (14):1738-1743

3 Simunovic N, Devereaux PJ, Sprague S, et al. Effect of early surgery after hip fracture on mortality and complications: systematic review and meta-analysis. CMAJ 2010;182(15):1609-1616

4 Panula J, Pihlajamäki H, Mattila VM, et al. Mortality and cause of death in hip fracture patients aged 65 or older: a populationbased study. BMC Musculoskelet Disord 2011;12:105

5 Schnell S, Friedman SM, Mendelson DA, Bingham KW, Kates SL. The 1-year mortality of patients treated in a hip fracture program for elders. Geriatr Orthop Surg Rehabil 2010;1(01):6-14
6 Parker M, Johansen A. Hip fracture. BMJ 2006;333(7557):27-30

7 Koval KJ, Skovron ML, Aharonoff GB, Meadows SE, Zuckerman JD. Ambulatory ability after hip fracture. A prospective study in geriatric patients. Clin Orthop Relat Res 1995;(310):150-159

8 Koval KJ, Zuckerman JD. Functional recovery after fracture of the hip. J Bone Joint Surg Am 1994;76(05):751-758

9 Bottle A, Aylin P. Mortality associated with delay in operation after hip fracture: observational study. BMJ 2006;332(7547):947-951

10 Gdalevich M, Cohen D, Yosef D, Tauber C. Morbidity and mortality after hip fracture: the impact of operative delay. Arch Orthop Trauma Surg 2004;124(05):334-340

11 Perez JV, Warwick DJ, Case CP, Bannister GC. Death after proximal femoral fracture-an autopsy study. Injury 1995;26(04):237-240

12 Novack V, Jotkowitz A, Etzion O, Porath A. Does delay in surgery after hip fracture lead to worse outcomes? A multicenter survey. Int J Qual Health Care 2007;19(03):170-176

13 Rogers FB, Shackford SR, Keller MS. Early fixation reduces morbidity and mortality in elderly patients with hip fractures from lowimpact falls. J Trauma 1995;39(02):261-265

14 Majumdar SR, Beaupre LA, Johnston DW, Dick DA, Cinats JG, Jiang HX. Lack of association between mortality and timing of surgical fixation in elderly patients with hip fracture: results of a retrospective population-based cohort study. Med Care 2006;44(06): 552-559

15 Grimes JP, Gregory PM, Noveck H, Butler MS, Carson JL. The effects of time-to-surgery on mortality and morbidity in patients following hip fracture. Am J Med 2002;112(09):702-709

16 Siegmeth AW, Gurusamy K, Parker MJ. Delay to surgery prolongs hospital stay in patients with fractures of the proximal femur.J Bone Joint Surg Br 2005;87(08):1123-1126

17 Franzo A, Simon G, Francescutti C. Mortality associated with delay in operation after hip fracture: ... but Italian data seem to contradict study findings. BMJ 2006;332(7549):1093

18 Khan SK, Kalra S, Khanna A, Thiruvengada MM, Parker MJ. Timing of surgery for hip fractures: a systematic review of 52 published studies involving 291,413 patients. Injury 2009;40 (07):692-697

19 Lefaivre KA, Macadam SA, Davidson DJ, Gandhi R, Chan H, Broekhuyse HM. Length of stay, mortality, morbidity and delay to surgery in hip fractures. JBone Joint Surg Br 2009;91(07): 922-927 